\title{
Idebenone protects against atherosclerosis in apolipoprotein E-deficient mice via activation of the Sirt3-SOD2-mtROS pathway
}

\section{Wei Jiang}

Shandong University Qilu Hospital

Hongzhi Geng

Shandong University Qilu Hospital

Xiaoqing LV

Shandong University Qilu Hospital

Jing Ma

Shandong University Qilu Hospital

Pengfei Lin

Shandong University Qilu Hospital

Chuanzhu Yan ( $\nabla$ czyan@sdu.edu.cn )

Shandong University Qilu Hospital

Original investigation

Keywords: Atherosclerosis, SIRT3-SOD2-mtROS pathway, idebenone, antioxidants, NLPR3, oxidative stress

Posted Date: February 14th, 2020

DOI: https://doi.org/10.21203/rs.2.23592/v1

License: (c) (i) This work is licensed under a Creative Commons Attribution 4.0 International License. Read Full License 


\section{Abstract}

Background: Atherosclerosis, which is a form of chronic aortic disease, results from the accumulation and aggregation of oxidized low density lipoprotein囚LDL囚in the vessel walls, the development of neointima, the formation of a fibrous cap, and the migration of immune cells to the damaged vascular endothelium. Recent studies have shown that mitochondrial function is closely associated with the development and progression of atherosclerosis. Idebenone functions as an electron carrier and antioxidant, and previous studies have shown that it effectively clears oxygen-free radicals. In the current study, we demonstrate that idebenone could protect against atherosclerosis using apolipoprotein Edeficient mice.

Methods: High-fat diet\HFD『and idebenone treatment with apolipoprotein E-deficient mice . A total of 60 mices were randomized into the following four groups: (1) HFD (2) idebenone-low dose (3) idebenonemedium dose and (4) idebenone-high dose for 16 weeks. The HUVECs were pretreated with endothelial cell medium in the presence or absence of $0.2 \mathrm{mM}$ idebenone working solution for $3 \mathrm{~h}$ followed by exposure to $10 \mu \mathrm{M}$ cholesterol for $24 \mathrm{~h}$. Proteomics analysis was performed between the HFD group $(n=3)$ and the high-dose idebenone group $(n=3$, concentration $=400 \mathrm{mg} / \mathrm{kg} / \mathrm{d})$.

Results: Compared with the HFD group, Idebenone can suppresses the formation of atherosclerotic plaques and increases the stability of atherosclerotic plaques in apoE-/- mice; Compared with the control group, Idebenone can protects against endothelial cell damage and inhibits the production of mtROS in cholesterol mediated HUVECs; Idebenone could effectively inhibit the development and progression of atherosclerosis and the injury of endothelial cells through the SIRT3-SOD2-mtROS pathway.

Conclusions: We showed that idebenone could act as a mitochondrial protective agent during the development and progression of atherosclerosis by inhibiting the activation of NLPR3 via the SIRT3SOD2-mtROS pathway. Idebenone may be a promising therapy for patients with atherosclerosis in the future, as it can improve mitochondrial dysfunction and inhibit oxidative stress.

\section{Background}

Atherosclerosis is the leading cause of morbidity and mortality in most developing and developed countries worldwide [1]. As a type of chronic inflammatory disease, atherosclerosis results from the aggregation of oxidized LDL in the vessel walls, the formation of a fibrous cap, the development of intimal hyperplasia, and the migration of immune cells to the damaged vascular endothelium [2]. Mitochondrial dysfunction has been increasingly associated with the evolution of atherosclerotic lesions due to the increased production of reactive oxygen species (ROS), which results in mitochondrial dysfunction and oxidative damage, along with impaired mitochondrial oxidative phosphorylation [3, 4]. Previously, Vasquez-Trincado et al. found that aortic wall samples from atherosclerotic patients displayed higher degrees of mitochondrial DNA damage than aortic samples from non-atherosclerotic donors [5]. In addition, mitochondrial DNA damage can lead to mitochondrial dysfunction through various 
physiological mechanisms, directly contributing to the formation and progression of atherosclerosis [6]. Therefore, mitochondrial dysfunction may be an important factor that promotes the initiation and progression of atherosclerosis.

Mitochondrial oxidative damage caused by the increased production of mitochondrial reactive oxygen species (mtROS) has become one of the significant mechanisms attributing to the formation of fatty plaques in the arteries, also known as atherogenesis [7, 8]. As a class of small molecules, mtROS play critical roles in several biological processes essential for cells and organisms to function. Any excess mtROS can be scavenged by the antioxidant defense system, including superoxide dismutase (SOD), catalase, and glutathione peroxidase [9ه10]. However, $1-2 \%$ of electrons leak out and produce superoxide free radicals, including mtROS during the oxidation phosphorylation process [11]. The overproduction of mtROS leads to the excessive oxidation of lipids and proteins, which can have significant effects on cell functioning [12]. Furthermore, the excessive production of mtROS induces mitochondrial dysfunction, endothelial cell damage, and aggregation of oxidized low-density lipoprotein (oxLDL) in smooth muscle cells and vascular endothelial cells, which can accelerate the progression of atherosclerosis [3凶13]. Moreover, mtROS may contribute to the inflammatory response in the progression of atherosclerosis via increased expression of certain inflammatory and adhesion factors [14囚15]. Hence, mtROS plays an important role in the inflammatory response of vascular endothelial cells, as well as the oxidation of cholesterol, which are now considered to be primary components of the pathophysiology of atherosclerosis.

Idebenone is a synthetic quinone structurally similar to coenzyme Q10 (CoQ10); however, it has less and shorter lipophilic tails than CoQ10. Idebenone is a novel type of antioxidant that effectively functions in hypoxic microenvironments [16]. By preventing lipid peroxidation and removing oxygen-derived free radicals, idebenone protects the membranes of cells and mitochondria from oxidative damage [17]. Therefore, the benefit of idebenone for delaying the development and progression of atherogenesis is of significant interest to the clinical community, especially considering its excellent safety profile.

In our previous study, we showed that idebenone could protect cells against mitochondrial damage and cell apoptosis caused by ox-LDL through GSK-3 $\beta / \beta$-catenin signaling in human umbilical vein endothelial cells (HUVECs) [18]. These findings indicate that idebenone could reduce endothelial cell injury caused by oxidative stress. In the present study, we investigated whether idebenone could protect against atherosclerosis in apolipoprotein E-deficient mice. In addition, we used functional analyses to explore potential mechanisms. Our findings indicated that idebenone could act as a mitochondrial protective agent in the initiation and progression of atherosclerosis by inhibiting the activation of NLPR3 through the Sirt3-Sod2-mtROS signaling pathway. Idebenone may be a promising drug in the prophylaxis or treatment of atherosclerotic lesion by improving the function of mitochondria, restraining the inflammatory responses, and inhibiting oxidative stress.

\section{Materials And Methods}


This study was approved by Brain Science Research Institute and the Ethics Committee from Qilu Hospital of Shandong University (Jinan, China).

\section{Reagents}

Endothelial cell medium (ECM), fetal bovine serum (FBS), and endothelial cell growth supplement (ECGS) were purchased from ScienCell Research Laboratories (Carlsbad, CA, USA). Cholesterol was obtained from MedChemExpress (HY-N0322, Monmouth Junction, NJ). Modified Masson's Trichrome Staining kits were purchased from Beijing Solarbio Science and Technology Co., Ltd. (Beijing, China). The mitochondrial membrane potential assay kit with JC-1, cell counting kit-8 (CCK-8) and SOD2 assay kit were purchased from Beyotime Institute of Biotechnology (Jiangsu, China). Microscale lipid peroxidation MDA assay kit was purchased from Jiancheng Bioengineering Institute (Nanjing, China). MitoSOX ${ }^{\mathrm{TM}}$ Red Mitochondrial Superoxide Indicator for live-cell imaging was purchased from Thermo Fisher Scientific (Waltham, MA, USA). The bicinchoninic acid (BCA) kit was purchased from Thermo Fisher Scientific (Waltham, MA, USA). Primary antibodies against Ac-SOD2 (ab137037), FOXO3A (ab12162), GAPDH (ab8245), $\beta$-actin (ab8226), TNF-a (ab1793), CD68 (ab125212), and a-SMA (ab32575) were obtained from Abcam (Cambridge, UK). The primary antibodies against Bcl-2 (2876), Bax (2772), Cleaved Caspase3 (9661), SIRT3 (2627), and NLRP3 (15101) were obtained from Cell Signaling Technologies (Beverly, MA, USA). The primary antibodies against IL-1 $\beta(N B 600-633)$ were obtained from Novus Biologicals (Colorado,USA). The primary antibody against Caspase-1 (22915-1-AP) was obtained from Proteintech Group (Manchester, UK).

\section{Drug and solution preparation}

We obtained idebenone powder from Qilu Pharmaceutical Co., Ltd. (Shandong, China). The idebenone, with a purity $>98 \%$, was dissolved in absolute ethyl alcohol to prepare a stock solution of $50 \mathrm{mM}$ for the in vitro experiments. For in vivo studies, idebenone was dissolved in corn oil for daily intragastric administration. A high-cholesterol and high-fat diet (15\% cocoa butter and $0.25 \%$ cholesterol) was purchased from Beijing Keao Xieli Limited Company (Beijing, China).

\section{Animal and tissue preparation}

A total of 60 apoE-/- mice (male, eight-week-old) were obtained from Beijing Vital River Laboratory Animal Technology Co., Ltd (Beijing, China). The mice were kept in a controlled environment with a constant temperature of $22 \pm 2{ }^{\circ} \mathrm{C}$ and humidity of $50-60 \%$. The mice were subjected to a $12 \mathrm{~h}$ light-dark cycle and had free access to food and water. The animal experimental protocol complied with the Animal Management Rules of the Chinese Ministry of Health and was approved by the Animal Care Committee of Shandong University.

To assess the stability of atherosclerotic plaques, all of the mice were given a high-fat diet (HFD), consisting of $15 \%$ cocoa butter and $0.25 \%$ cholesterol, for the duration of the study. One week after starting the high-fat diet, the mice were randomly divided into four groups ( $n=15$ per group) for this study, including the HFD group (equal volume of corn oil as the three drug treatment groups), low-dose 
idebenone group (IDE-L), medium-dose idebenone group (IDE-M), and high-dose idebenone group (IDE-H). The mice were given $0.1,0.2$, or $0.4 \mathrm{~g} / \mathrm{kg} / \mathrm{d}$ of idebenone in the IDE-L, IDE-M, and IDE-H groups, respectively.

All animals were weighed at 16 weeks of age. The mice were fasted for $4 \mathrm{~h}$ before blood samples were harvested for the lipid analysis and tissue assays. At the end of the experiment, the mice were euthanized by $10 \%$ chloral hydrate $(0.01 \mathrm{ml} / \mathrm{g})$, and the aorta, brain, and liver tissues were extracted, flash-frozen with liquid nitrogen, and stored at $-80{ }^{\circ} \mathrm{C}$ for future experiments. Another group of mice was anesthetized with $10 \%$ chloral hydrate $(0.001 \mathrm{ml} / \mathrm{g})$, and blood samples were collected. Next, the cardiovascular system was rapidly perfused with $0.9 \%$ saline, followed by $4 \%$ paraformaldehyde, and the mouse died during perfusion ultimately. The collected specimens were fixed with $4 \%$ paraformaldehyde. The aortic roots were embedded in optimum cutting temperature (OCT) compound for cryosectioning. Serial crosssections of $5-\mu \mathrm{m}$ thickness were obtained and stored at $-20^{\circ} \mathrm{C}$ for future immunohistochemistry studies.

\section{Cell culture}

Human umbilical vein endothelial cells (HUVECs) were obtained from the umbilical cords of healthy human mothers following deliveries. The freshly isolated HUVECs from the human umbilical cord were cultured with ECM supplemented with $10 \%$ FBS, $1 \%$ penicillin/streptomycin, and ECGS in a humidified incubator at $37{ }^{\circ} \mathrm{C}$ with $5 \% \mathrm{CO}_{2}$. The HUVECs displayed a cobblestone morphology and stained positively for common endothelial cell marker, known as the Von Willebrand factor (VWF). The cells were passaged four to eight times before they were used for experiments. Idebenone was dissolved in absolute ethyl alcohol to generate a working solution of $0.2 \mathrm{mM}$. The HUVECs were pretreated with ECM in the presence or absence of the idebenone working solution for $3 \mathrm{~h}$ to reach $50-60 \%$ confluency, followed by exposure to $10 \mu \mathrm{M}$ cholesterol for $24 \mathrm{~h}$ while maintaining the same concentration of idebenone.

\section{Western blot analysis}

HUVECs and aorta tissue samples were lysed using radio-immunoprecipitation assay (RIPA) lysis buffer and subjected to Western blot as described previously [18]. Briefly, the pre-stained protein ladder and equal amounts of protein were separated on $8-15 \%$ sodium dodecyl sulfate-polyacrylamide gels by electrophoresis, followed by transfer to methanol-activated polyvinylidene fluoride membranes. To block non-specific antigens, the membranes were incubated in $5 \%$ skimmed milk for 60 min at $37{ }^{\circ} \mathrm{C}$. Next, the blots were incubated with primary antibodies, previously identified in the reagents section, at dilutions of 1:500 to $1: 1,000$ overnight at $4{ }^{\circ} \mathrm{C}$. After incubating with the primary antibodies, the membranes were washed with TBST three-times and incubated with horseradish peroxidase (HRP)-secondary antibodies for 60 min. Protein bands were visualized with the Millipore ECL Plus reagent and imaged on Tanon-5500 Imaging Analysis System. The intensities of the bands were quantified using Image J software.

\section{Histopathological analysis}

The en face aorta was stained with oil-red $O$ to assess the overall burden and distribution of atherosclerosis as previously described. The aortic roots, which are predilection sites of atherosclerosis, 
were cut into 5- $\mu \mathrm{m}$ thick cross-sections, and stained with hematoxylin and eosin (H\&E), oil-red O, Sirius Red, and Masson's trichrome stain. The sections were reacted with primary antibodies against CD68, smooth muscle cells (SMCs), and tumor necrosis factor- $a$ (TNF-a). Oil-red 0 stained the lipids in the plaque, while the Picrosirius red stain and Masson's stain were used to stain collagen.

Immunohistochemical staining was performed in accordance with standard protocols from the Shandong Province Joint Key Laboratory of Translational Cardiovascular Medicine of Qilu Hospital. In brief, the aortic root sections were incubated with $3 \%$ hydrogen peroxide for reducing endogenous peroxidase activity and blocked with $5 \%$ goat serum for 30 min at room temperature. Next, the sections were incubated with primary antibodies overnight at $4{ }^{\circ} \mathrm{C}$, followed by washing three times with phosphate-buffered saline (PBS). After washing the slides, the aortic root sections were incubated with the secondary antibodies for $60 \mathrm{~min}$ at room temperature. A 3'3-diaminobenzidine (DAB) kit was used for immunohistochemical staining. The primary antibodies were anti-CD68 (1:150, Abcam, Cambridge, UK), a-smooth muscle actin (a-SMA, 1:200, Abcam, Cambridge, UK), TNF-a (1:200, Abcam, Cambridge, UK). Histological and immunohistochemical stained sections were analyzed using Image-Pro Plus 6.0 (IPP 6.0, Media Cybernetics, Silver Springs, MD, USA). The en face analysis of the aorta sections was performed as previously described. Each picture was counted three times, and the average value was adopted as the final result. All measurements were performed under the same conditions to ensure consistency.

\section{Protein extraction, identification, and analysis}

Vascular tissue samples of the HFD group and IDE-H group were sonicated three times on ice using a high-intensity ultrasonic processor (Ningbo Scientz Biotechnology Co., Ltd., Zhejiang, China) in lysis buffer, consisting of $8 \mathrm{M}$ urea and $1 \%$ protease inhibitor cocktail. The samples were centrifuged at $12,000 \mathrm{~g}$ and $4{ }^{\circ} \mathrm{C}$ for $10 \mathrm{~min}$. Finally, the supernatant was collected, and the protein concentration was determined using the BCA kit, according to the manufacturer's instructions.

For digestion, the protein solution was reduced with $5 \mathrm{mM}$ dithiothreitol for $30 \mathrm{~min}$ at $56^{\circ} \mathrm{C}$ and alkylated with $11 \mathrm{mM}$ iodoacetamide for $15 \mathrm{~min}$ at room temperature in darkness. The protein sample was then diluted by adding $100 \mathrm{mM}$ triethylamine-carbonic acid buffer (TEAB). Finally, trypsin was added at a 1:50 trypsin-to-protein mass ratio for the first digestion overnight and 1:100 trypsin-to-protein mass ratio for a second 4 h-digestion.

After the trypsin digestion, the peptide was desalted with the Strata X C18 SPE column (Phenomenex, Torrance, CA, USA) and vacuum dried. The peptide was reconstituted in $0.5 \mathrm{M}$ TEAB and processed according to the manufacturer's protocol for the TMT kit/iTRAQ kit. Briefly, one unit of the TMT/iTRAQ reagent was thawed and reconstituted in acetonitrile. The peptide mixtures were then incubated for $2 \mathrm{~h}$ at room temperature and pooled, desalted, and dried by vacuum centrifugation.

\section{Cell viability assay}

For the CCK-8 assay, $3 \times 10^{3}$ HUVECs were seeded into 96-well culture plates with ECM for $24 \mathrm{~h}$. Next, the media was changed with fresh ECM containing $0.2 \mathrm{mM}$ of the idebenone working solution. After $3 \mathrm{~h}$, the 
HUVECs were exposed to $10 \mu \mathrm{M}$ of cholesterol for an additional $24 \mathrm{~h}$ without altering the concentration of idebenone. After co-cultivating idebenone and cholesterol for $24 \mathrm{~h}, 10 \mathrm{ml}$ of the CCK-8 assay solution was added to the cells and incubated for $2 \mathrm{~h}$ at $37^{\circ} \mathrm{C}$. The cell viability was detected by EnSpire ${ }^{\circledR}$ Multimode Plate Reader (Perkin Elmer, Waltham, MA, USA) by recording the optical density at $450 \mathrm{~nm}$. Cell viability in the control groups was considered $100 \%$, and each experiment was performed in triplicate.

\section{JC-1 (MitoScreen) and MitoSOX assays}

The depolarization of the mitochondrial membrane potential (MMP) was determined using the JC-1 kit. The cells were seeded in 96 well plates at $3 \times 10^{3}$ cells per well for $24 \mathrm{~h}$. Next, fresh ECM containing $0.2 \mathrm{mM}$ of the idebenone working solution was added to the cells. After $3 \mathrm{~h}$, the HUVECs were exposed to $10 \mu \mathrm{M}$ of cholesterol for an additional $24 \mathrm{~h}$ without altering the concentration of idebenone. After idebenone and cholesterol were co-cultured for $24 \mathrm{~h}$, the HUVECs were washed three times with PBS. For the MitoScreen assay, $100 \mathrm{ml}$ of the JC-1 working solution was added to each well. The JC-1 working solution contained the JC-1 stock solution and buffered solution at a 1:100 ratio. Finally, all samples were incubated at $37{ }^{\circ} \mathrm{C}$ for 15 minutes, before the cells were washed with the JC-1 staining buffer in duplicate and assessed on a microplate reader. To measure mtROS levels with the MitoSOX red stain assay, the HUVECs were incubated with $5 \mu \mathrm{M}$ of MitoSOX red and MitoTracker at $37^{\circ} \mathrm{C}$ for $15 \mathrm{~min}$. A fluorescence microscope was used to detect the signals with an excitation and emission wavelengths of 510 and $580 \mathrm{~nm}$, respectively (SP8, Leica Biosystems, Wetzlar, Germany).

\section{Measurement of MDA and SOD activity}

HUVECs from three groups were washed with PBS, lysed in a lysis buffer and scraped from the plates. The lysates were centrifuged at $12,000 \mathrm{~g}$ for $15 \mathrm{~min} 4{ }^{\circ} \mathrm{C}$. Total proteins were then extracted with a RIPA lysis buffer and the protein concentrations were determined by using the BCA method. Subsequently, the supernatants were collected and the concentration of malonaldehyde (MDA) and the activities of SOD2 in the lysates were examined by using the microscale lipid peroxidation MDA assay kit and SOD2 assay kit.

\section{Statistical analysis}

Numerical data were expressed as means \pm SD based on three or more independent experiments. Differences between groups were determined and analyzed using the one-way analysis of variance (ANOVA) with Fisher's Least Significant Different (LSD) using the SPSS 16.0 software package (IBM, Chicago, IL, USA). P-values $<0.05$ were considered to be statistically significant.

\section{Results}

\section{Bodyweight, glucose concentration, and serum lipid profiles levels are not altered by idebenone treatment}

Table 1 shows the body weights, blood lipid analyses, and blood glucose levels of mice from this study. The related data analysis indicated that there were no significant differences in blood lipid levels, 
including triglycerides (TG), total cholesterol (TC), high-density lipoprotein cholesterol (HDL-C), or lowdensity lipoprotein cholesterol (LDL-C) between the four groups of apoE-/- mice (Table 1). In addition, idebenone treatment did not cause any significant changes in body weight or blood glucose levels, as compared with the HFD group.

Table 1

Bodyweight, glucose concentration and serum lipid profiles levels

\begin{tabular}{|c|c|c|c|c|c|c|}
\hline Groups & $\begin{array}{l}\text { BW } \\
(g)\end{array}$ & $\begin{array}{l}\text { Glucose } \\
(\mathrm{mmol} / \mathrm{L})\end{array}$ & $\begin{array}{l}\text { TC } \\
(\mathrm{mmol} / \mathrm{L})\end{array}$ & $\begin{array}{l}\text { TG } \\
(\mathrm{mmol} / \mathrm{L})\end{array}$ & $\begin{array}{l}\text { LDL - C } \\
(\mathrm{mmol} / \mathrm{L})\end{array}$ & $\begin{array}{l}\mathrm{HDL}-\mathrm{C} \\
(\mathrm{mmol} / \mathrm{L})\end{array}$ \\
\hline HFD & $35.82 \pm 0.26$ & $8.36 \pm 0.15$ & $25.43 \pm 0.31$ & $1.83 \pm 0.21$ & $3.52 \pm 0.09$ & $7.80 \pm 0.13$ \\
\hline IDE - L & $35.38 \pm 0.24$ & $8.08 \pm 0.15$ & $25.22 \pm 0.29$ & $1.84 \pm 0.15$ & $3.48 \pm 1.00$ & $7.82 \pm 0.17$ \\
\hline IDE - M & $35.20 \pm 0.19$ & $8.04 \pm 0.16$ & $24.86 \pm 0.35$ & $1.82 \pm 0.16$ & $3.46 \pm 0.09$ & $7.98 \pm 0.18$ \\
\hline IDE - H & $35.13 \pm 0.20$ & $7.95 \pm 0.13$ & $24.83 \pm 0.27$ & $1.78 \pm 0.08$ & $3.44 \pm 0.08$ & $8.07 \pm 0.14$ \\
\hline$P$ value & ns & ns & ns & ns & ns & ns \\
\hline \multicolumn{7}{|c|}{$\begin{array}{l}\text { Data are expressed as the mean } \pm \text { SEM. HFD: high fat diet, IDE: idebenone, BW: body weight, TC: total } \\
\text { cholesterol, TG: triglycerides, LDL-C: low-density lipoprotein cholesterol, HDL-C: high-density } \\
\text { lipoprotein cholesterol, ns: not significant. }\end{array}$} \\
\hline
\end{tabular}

To verify the inhibitory effect of idebenone in atherosclerosis, three different concentrations of idebenone were used for the intragastric administrations in apoE-/- mice, which were fed high-cholesterol diets since the age of four months. The en face lesion analysis showed that the relative lesion area was significantly reduced in the three treatment groups, as compared with the HFD group, especially the high-dose idebenone group (Fig. 1A and 1C). Besides, the cross-sectional plaque area of the aortic sinus was reduced significantly in the three treatment groups when compared with the HFD group, especially in the idebenone-H group (Fig. 1B and 1D). Accordingly, our study demonstrated that idebenone could attenuate the formation of atherosclerotic plaques in the entire aorta and aortic root of apoE-/- mice.

\section{Idebenone treatment increases the stability of atherosclerotic plaques in apoE-/- mice}

To further confirm whether idebenone could alter the components of atherosclerotic plaques, we performed histology and immunohistochemistry staining of the aortic root sections. The lipid and macrophage content in atherosclerotic plaques decreased significantly in the three treatment groups, as compared with the HFD group, especially in the idebenone-H group (Fig. 2A, 2E, 2H, and 2K). The collagen content in the plaques was significantly higher in the three treatment groups than the HFD group (Fig. 2B, 
2C, 2D, 2I, and 2J). Similarly, the plaque collagen content of SMCs was significantly increased in the three treatment groups, as compared with the HFD group (Fig. 2F and 2L). Furthermore, idebenone could suppress the expression of TNF-a in a dose-dependent manner (Fig. $2 \mathrm{G}$ and $2 \mathrm{M}$ ).

These findings demonstrate that idebenone treatment dose-dependently changes the composition of atherosclerotic plaques to form more stabilized phenotypes. We confirmed alterations in the composition of the plaques, which hinted that idebenone could effectively change the stability of intravascular plaques and inhibit the development and progression of atherosclerosis.

\section{Proteomic analysis between the HFD group and the high- dose idebenone group}

To further study how idebenone protects against atherosclerosis and how the effect is modulated, a proteomics analysis was performed between the HFD group $(n=3)$ and the high-dose idebenone group ( $n$ $=3$, concentration $=400 \mathrm{mg} / \mathrm{kg} / \mathrm{d}$ ), as the high-dose group showed the most obvious phenotypic differences between the treatment groups.

From the Volcano plot of the distribution of differentially expressed proteins in the different groups, 351 upregulated and 379 downregulated proteins were found to be significantly altered, as determined with a threshold of 1.3 (Fig. 3A). The differentially expressed proteins were primarily located in mitochondria, extracellular matrix, and cytoplasm in the subcellular localization chart (Fig. 3B). Next, Gene Ontology (GO) enrichment bubble plots of the differentially expressed proteins in the three categories and comprehensive heatmaps for the cluster analysis are shown in Fig. 3C-J. The clusters of orthologous groups of proteins (COG)/eukaryotic orthologous groups (KOG) functional classification chart of differentially expressed proteins was primarily involved in five processes, including energy production and conversion, amino acid transport and metabolism, lipid transport and metabolism, general function predictions, and signal transduction (Fig. 1S, Supplementary Materials). As idebenone is a mitochondrial protectant and antioxidant, more attention was given to the differentially expressed proteins associated with mitochondrial function. To assess the proteomic analysis, only proteins related to the development of atherosclerosis and the function of mitochondrial and oxidative stress were studied. From the proteomics and Western blot analyses, three proteins were detected: SIRT3, AC-SOD2, and NLRP3 (Fig. 3K-N). The six samples used for western blot were the same as those used for the proteomics analysis to ensure consistency between the studies. From the three proteins detected by both analyses, SOD2 is primarily activated by the deacetylation of specific conserved lysine residues and catalyzed by SIRT3, which plays an essential role in the clearance of the mtROS that could induce NLRP3-related inflammatory responses. Hence, idebenone likely protect against the initiation and progression of atherosclerosis through modulation of the SIRT3-SOD2-mtROS signaling pathway.

\section{Idebenone activates the SIRT3-SOD2-mtROS pathway and inhibits NLRP3-induced inflammatory responses in apoE-/- mice}


The involvement of the SIRT3-SOD2-mtROS-dependent pathway was further verified in the three idebenone treatment groups and the HFD group of apoE-/- mice. In addition, the expression levels of SIRT3, FOXO3A, and AC-SOD2 were detected by western blot in aortic tissue samples from the different groups. From these experiments, idebenone was found to significantly upregulate the expression of SIRT3 (Fig. 4A-B), AC-SOD2 (Fig. 4A and 4D), and FOXO3A (Fig. 4A and 4C) in all treatment groups as compared with the HFD group. In a previous study, excess mtROS production caused by mitochondrial dysfunction could activate NLRP3, which would lead to the release of several inflammatory cytokines, such as caspase-1 (p20), and trigger a substantial inflammatory response. From the Western blot analysis, NLRP3 and caspase-1 (p20) were downregulated in the aortic tissues of apoE-/- mice when pretreated with idebenone in the three treatment groups (Fig. 4A, 4E, and 4F). NLRP3 can activate procaspase- 1 and ultimately lead to the processing and secretion of the pro-inflammatory cytokine IL-1 $\beta$. Downregulation in IL-1 $\beta$ were found between the different groups in the current study, as there were no changes in procaspase-1 expression after the mice were treated with idebenone (Fig. 4A and 4G). These findings further demonstrate that the protective effects of idebenone against atherosclerotic plaques are likely dependent on the SIRT3-SOD2-mtROS pathway and the reduction of the inflammatory response triggered by the activation of the NLRP3 inflammasome.

\section{Idebenone protects against endothelial cell damage and inhibits the production of mtROS in cholesterol mediated HUVECs}

The protective effects against endothelial cell damage and the relationship between idebenone and ROS generation were assessed next, especially mtROS in the cholesterol mediated HUVECs. Cholesterol was used at its optimal concentration of $10 \mu \mathrm{M}$, which can cause apoptosis of endothelial cells. We investigated that cholesterol significantly decreased the expression of $\mathrm{Bcl}-2$ and increased the expression of Bax and the relevant data show that pretreatment with idebenone suppressed the down regulation of Bcl-2 and up regulation of Bax in HUVECs exposed to cholesterol (Fig. 5C, 5D and 5E). Furthermore, caspase- 3 is a key factor in the initiation of apoptosis. As shown in Fig. $5 \mathrm{C}$ and $5 \mathrm{~F}$, cholesterol significantly increased the protein levels of caspase-3 in cultured HUVECs, which could be suppressed by idebenone pre-treatment .

Then, the effects of pre-treatment with $0.2 \mu \mathrm{M}$ of idebenone on cell viability were examined in cholesterolmediated HUVECs using the CCK-8 assay. After $24 \mathrm{~h}$ of incubation, the HUVECs with $10 \mu \mathrm{M}$ of cholesterol caused noticeable changes in cell shrinkage, suspension, and retraction, as compared with the control group. However, $3 \mathrm{~h}$ of pre-treatment with $0.2 \mathrm{mM}$ idebenone prior to cholesterol significantly increase cell viability (Fig. 5G). These results indicate that idebenone could effectively prevent the cholesterol-induced damage to endothelial cells.

Similar to the experimental methods described above, after cholesterol and idebenone co-cultivation for $24 \mathrm{~h}$, the mitochondrial membrane potential in the HUVECs was detected with the JC-1 kit. The JC- 1 dye 
is commonly used to detect mitochondrial potential, as it preferentially enters the mitochondria due to its highly negative MMP. The ratio of red/green fluorescence depends on the membrane potential, as JC-1 accumulates in polarized mitochondria with negative MMPs and becomes red. However, when the MMP

is decreased, the $\mathrm{JC}-1$ aggregates turn into monomer and appear green. Hence, MMP was assessed using the JC-1 dye to detect both aggregates (red) and monomers (green). The fluorescence levels were acquired using a microplate system with excitation wavelengths of $514 \mathrm{~nm}$ and $585 \mathrm{~nm}$ for both green and red fluorescence, respectively. The fluorescence intensity was analyzed with ImageJ software. Cholesterol significantly decreased the ratio of aggregates to monomers, which was increased by pretreatment with idebenone (Fig. 5H). Finally, HUVECs pretreated with different concentrations of idebenone were pre-stained with MitoSOX and MitoTracker dye for $15 \mathrm{~min}$ and revealed colocalization of the MitoSOX (Red) and MitoTracker (Green) stains. MitoSOX red is a highly selective fluorescent probe for the detection of mtROS generated within the mitochondria. Furthermore, the images of HUVECs revealed increased fluorescence in the mitochondria of those cells treated with cholesterol under a confocal laser scanning microscope, and suppressed fluorescence in the mitochondria of cells treated with cholesterol and idebenone (Fig. 5A and 5B). These findings provide additional evidence suggesting that idebenone can inhibit endothelial mtROS generation.

Incubation of HUVECs with $10 \mu \mathrm{M}$ cholesterol for $24 \mathrm{~h}$ caused the significant increase of MDA content and the marked decrease of SOD2 activity, compared with those of cells control group. Pretreated with $0.2 \mathrm{mM}$ idebenone for $3 \mathrm{~h}$ markedly attenuated the increase in MDA content and decreased SOD2 activities in response to cholesterol respectively (Fig. $5 \mathrm{I}$ and $5 \mathrm{~J}$ ).

\section{Idebenone inhibits cholesterol-induced injury of HUVECs by modulating SIRT3 activation and NLRP3-related inflammatory reactions}

In the next experiment, it was determined whether idebenone could protect endothelial cells through the SIRT3-SOD2 pathway and NLRP3-related inflammatory reactions in vitro. The downregulation of NLRP3 (Fig. 6A and 6E) and upregulation of SIRT3 (Fig. 6A and 6B), AC-SOD2 (Fig. 6A and 6D), and FOXO3A (Fig. $6 \mathrm{~A}$ and $6 \mathrm{C}$ ) were detected in cholesterol damaged HUVECs after pre-treatment with $0.2 \mu \mathrm{M}$ of idebenone.

Caspase-1 (p20) is a critical factor in inflammatory responses and apoptosis. In this study, cholesterol significantly increased the protein levels of caspase-1 (p20) in cultured HUVECs, which could be suppressed by idebenone pre-treatment (Fig. 6A and 6F). The NLRP3 inflammasome is an IL-1ßfamily cytokine-activating protein complex consisting of inactive procaspase-1. Decreased expression of IL$1 \beta$ were detected between the three treatment groups (Fig. $6 \mathrm{~A}$ and $6 \mathrm{G}$ ). The in vitro findings are consistent with the in vivo studies, which showed that activation of the SIRT3-SOD2 mitochondrial pathway, inhibition of the associated inflammatory responses, and scavenging of mtROS (Fig. 5A and 5D) might play vital roles in the protection of cholesterol-induced injury to HUVECs with idebenone treatment. 


\section{Discussion}

According to previous studies, oxLDL and cholesterol can induce the production of ROS, which leads to mitochondrial dysfunction, cell apoptosis, and oxidative stress in the arteries [7]. The mitochondria are the primary site of ROS production and accumulation in cells, and impairment of mitochondrial function can lead to excess ROS production, which results in vascular endothelial cell dysfunction and atherosclerosis $[19,20]$. Previously, Ballinger SW et al. demonstrated that the accumulation of mtROS and DNA damage can increase the progression of atherosclerotic lesions in human arterial specimens and mouse models of atherosclerosis [21]. In addition, Yu E et al. showed that mtDNA damage in circulating cells and vessel walls was indicative of a higher risk of atherosclerosis [22]. In summary, mitochondria play a vital role in the connection of atherosclerosis with oxidative stress, inflammatory responses, and endothelial dysfunction. Hence, the early treatment of mitochondrial dysfunction is of vital importance in the prevention and treatment of atherosclerosis.

Most mitochondrial protective agents have been shown to modulate mitochondrial dysfunction and prevent atherogenesis. Mitoquidone, which is a new type of ROS scavenger that primarily acts on mtROS, could diminish the formation of free radicals without affecting oxidative phosphorylation or mitochondrial function. In mouse models, mitoquidone was shown to decrease cell proliferation and macrophage content in arterial plaques, while also inhibiting certain characteristics of metabolic syndromes [23]. As another ROS scavenger, idebenone is a short-chain benzoquinone with a structure similar to that of CoQ10 [24]. The pharmacological properties of idebenone have been shown to treat neurodegenerative diseases, such as aged dementia, Parkinson's disease, and Friedreich's ataxia [25]. In our previous study, we demonstrated that idebenone could prevent oxLDL-induced mitochondrial dysfunction in vascular endothelial cells [18]. Subsequently, we confirmed that idebenone could effectively inhibit the development and progression of atherosclerosis both in vivo and in vitro.

Idebenone functions as an antioxidant and electron carrier, which are effective in clearing oxygen-free radicals [26]. In previous studies, idebenone was shown to inhibit complex I in the electron transport chain (ETC), while it also can contribute electrons to complex III[27, 28]. In addition, some studies have reported that the idebenone-dependent metabolic pathways could transfer equal amounts of energy directly from the cytoplasm into the mitochondrial respiratory chain (MRC) [29]. Since idebenone can easily cross the blood-brain barrier, it can readily be broken down into its metabolic products, including QS-10, QS-6, and QS-4 [30]. QS-10 is an electron acceptor with a strong affinity to NQ01, which is a key factor in protecting against oxidative stress and inhibiting the development of atherosclerosis [30]. Additionally, pre-treatment of cells with idebenone has been shown to attenuate the production of pro-inflammatory factors through the inhibition of the MAPK and NF-KB signaling pathways, which are important for promoting atherosclerosis [31]. Beside, idebenone is a known PPARa ligand that can protect endothelial cells and stabilize fatty plaques through various mechanisms [24]. Lastly, idebenone has an excellent safety profile in patients [32]. 
Our study demonstrated that idebenone could inhibit the development and progression of atherosclerosis in apolipoprotein E-deficient (apoE-/-) mice. Morphological and histological analyses revealed a reduction in the plaque burden of apoE-/- mice treated with idebenone, as compared with the HFD group. Furthermore, the intravascular plaque size was significantly reduced, and the composition of the plaque was stabilized in the three idebenone treatment groups.

We performed a proteomic analysis between the HFD group and the IDE-H group to explore the related molecular mechanisms. We determined that idebenone may function by increasing the expression of SIRT3 and SOD2, which inhibits endothelial mtROS generation and reduces NLRP3-related inflammatory responses. Based on our research, we suspect that idebenone can impact the development of atherosclerosis in two manners. First, as a mitochondrial protectant, idebenone can deliver leaked electrons and reduce the generation of mtROS; meanwhile, idebenone can produce a systemic antioxidant effect by minimizing the lipid peroxidation. LDL and mtROS can directly activate the NLRP3 inflammasome [33], thereby, idebenone avoide the initiation of the inflammatory cascade. Secondly, idebenone could increase intracellular SIRT3 levels, which can alter the acetylation of mitochondrial antioxidant enzymes including glutathione peroxidase, isocitrate dehydrogenase 2, and SOD2. In addition, SIRT3 can also regulating the expression of SOD2 by activating the transcription factor FOXO3A, which impacts its ability to scavenge mtROS thoroughly and suppress the NLRP3 inflammasome [34].

SIRTs are a family of sirtuins, which are NAD+-dependent histone deacetylases [35]. Activation of SIRTs appears to have beneficial effects for anti-oxygenation and lipid metabolism [36]. Sirtuin3 (SIRT3), one of the antioxidants of the Sir2 family, plays a vital role in the regulation of mitochondrial metabolism through the removal of excess mtROS and activation of specific metabolic enzymes in the mitochondria [37]. SIRT3 is a substrate involved in a variety of biological processes, such as mitochondrial membrane potential maintenance, electron transport chain flux, mitochondrial dynamics, energy metabolism, and the production and clearance of mtROS [38]. Dysfunctional mitochondria lead to the development of certain atherosclerotic diseases, such as myocardial infarction, through the inhibition of SIRT3 [39]. SIRT3 can deacetylate a number of mitochondrial-related proteins, including SOD2, which can limit the accumulation of mtROS in the mitochondria [40]. SIRT3 directly combines with and deacetylates SOD2, which then strengthens the activity of SOD2 and subsequently impacts mtROS homeostatic function [41]. Furthermore, excessive mtROS can stimulate the formation of the NLRP3 inflammasome, which in turn, triggers a series of inflammatory reactions $[42,43]$. The NLRP3-related inflammasome is a protein complex consisting of the inactive procaspase-1, adaptor protein apoptotic speck-like protein, and the pattern recognition receptor NLRP3. The NLRP3 inflammasome can activate IL-1 $\beta$ cytokines and participate in the inflammatory response and natural immunity [44]. The NLRP3 inflammasome is a vital inflammation signaling molecule that can be activated by various molecular mechanisms, including mtROS. As such, mtROS could stimulate the assembly of the NLRP3 inflammasome complex, thereby stimulating the secretion of the inflammatory cytokines, IL-1 $\beta$ and IL-18, along with the activation of caspase- $1[45 \llbracket 46]$. In the carotid atherosclerotic plaques of patients undergoing endarterectomy, there is 
increased expression of NLRP3, caspase-1 (p20), IL-1 $\beta$, and IL-18, all of which are higher in vulnerable plaques than stabilized plaques [47]. Inflammatory cells play a leading role in early atherosclerotic lesions, as they accelerate the formation of plaques through effector molecules, which results in the activation of inflammation and induction of cardiovascular disease [48囚49]. The physiological effects of the SIRT3-SOD2-NLRP3 pathway were previously shown in dysfunctional endothelial cells of the aorta, providing a potential direction in the prevention of cardiovascular diseases associated with atherosclerosis [38].

The possible pathogenic mechanisms were further explored by inhibiting the activation of the NLRP3 inflammasome with idebenone [50]. Inflammasome activators like cholesterol can enhance the production of mtROS, which reinforces the interaction of NLRP3 and thioredoxin interacting protein, and the activation of the NLRP3 inflammasome. Besides, mtROS scavengers can inhibit the activation of the NLRP3-related inflammasome[51,52]. The production of intracellular oxygen free radicals mostly comes from mitochondria. In our experiments, we use MitoSOX red to evaluate mtROS generation, which could differentiate the generation of ROS from the cytoplasm and mitochondria. As expected, idebenone treatment suppressed the red fluorescence in the mitochondria of HUVECs pretreated with cholesterol, indicating that idebenone could inhibit the generation of mtROS to a significant extent in our study. In addition, idebenone mitigated mtROS production as a specific mtROS scavenger [53], therefore inhibiting mtROS-induced activation of the NLRP3 inflammasome and ultimately attenuating the intra-plaque inflammatory reaction. In general, activation of the NLRP3 inflammasome in vascular endotheliocytes by cholesterol may be an important initial part of endothelial cell injury, and dysfunction of the endothelial cells through the development of atherosclerosis may be mediated through NLRP3 inflammasome-driven signal pathways. Our results demonstrate that idebenone can affect the development of atherosclerotic plaque by inhibiting NLRP3-inflammatory pathways caused by the excessive generation of mtROS, which provide theoretical support for a new drug target in the future.

\section{Conclusion}

Taken together, our findings indicate that idebenone treatment could mitigate the formation of plaques. Hence, it may play a critical role in atherosclerotic vulnerable plaque stabilization in apolipoprotein Edeficient mice. In vitro experiments demonstrated that idebenone protected mitochondria function, cleared mitochondrial oxygen free radicals, and blocked the cascade of inflammatory reactions. Idebenone could act as a protective agent against atherosclerosis through the possible SIRT3-SOD2mtROS pathway, which can inhibit the activation of NLPR3. In summary, considering the fact that idebenone has been used in clinical trials without reports of serious adverse effects, we believe that idebenone might be a promising agent for the prevention of atherosclerosis. More randomized controlled trials are needed to verify our findings in the future.

\section{Abbreviations}




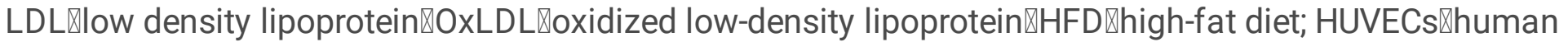
umbilical vein endothelial cells $₫ R O S \otimes$ reactive oxygen species $\triangle m t R O S \otimes$ mitochondrial reactive oxygen

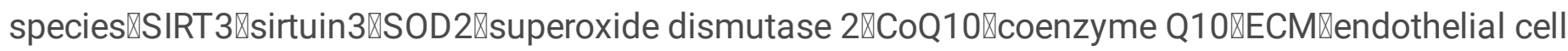

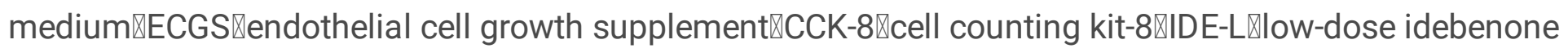

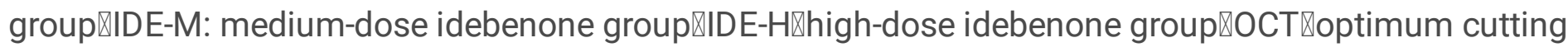

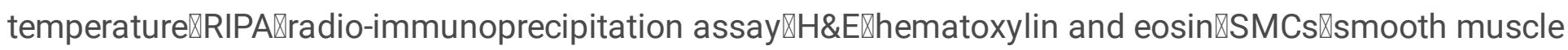

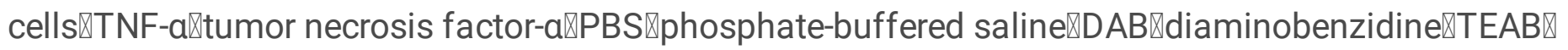

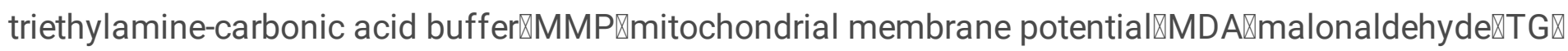

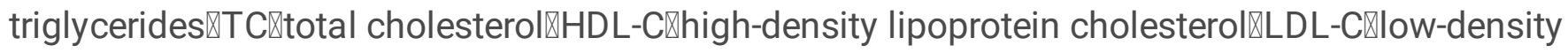

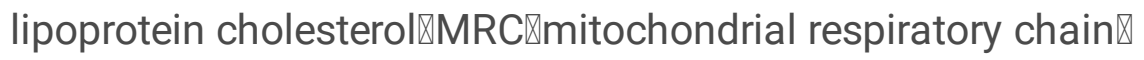

\section{Declarations}

\section{Ethics approval and consent to participate}

This study was approved by Brain Science Research Institute and the Ethics Committee from Qilu Hospital of Shandong University (Jinan, China).

\section{Consent for publication}

Written informed consent for publication was obtained from all participants.

\section{Availability of data and materials}

The used and/or analyzed datasets are available from the corresponding author on reasonable request.

\section{Competing interests}

The authors declare there are no known conflicts of interest associated with this publication, and there has been no significant financial support for this work that could have influenced its outcome.

\section{Funding}

This work was supported by the National Science Foundation Research Grant (Grant No. 81671235) and the Taishan Scholars Program of Shandong Province.

\section{Authors' contributions}

Wei Jiang, Pengfei Lin, and Chuanzhu Yan conducted the conception and design of the study, acquisitionand interpretation of data, drafting the article, final approval of the versionto be published.

\section{Acknowledgements}

We thank the State and Shandong Province Joint Key Laboratory of Translational Cardiovascular Medicine for appropriate advices about the animal experimental protocol. 


\section{References}

[1] S. Pant, A. Deshmukh, G.S. Gurumurthy, N.V. Pothineni, T.E. Watts, F. Romeo, J.L. Mehta, Inflammation and atherosclerosis--revisited, J Cardiovasc Pharmacol Ther 19(2) (2014) 170-8.

[2] F. Schaftenaar, V. Frodermann, J. Kuiper, E. Lutgens, Atherosclerosis: the interplay between lipids and immune cells, Curr Opin Lipidol 27(3) (2016) 209-15.

[3] U. Forstermann, N. Xia, H. Li, Roles of Vascular Oxidative Stress and Nitric Oxide in the Pathogenesis

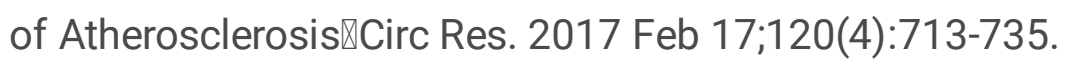

[4] J.L. Fetterman, M. Holbrook, D.G. Westbrook, J.A. Brown, K.P. Feeley, R. Breton-Romero, E.A. Linder, B.D. Berk, R.M. Weisbrod, M.E. Widlansky, N. Gokce, S.W. Ballinger, N.M. Hamburg, Mitochondrial DNA damage and vascular function in patients with diabetes mellitus and atherosclerotic cardiovascular disease, Cardiovasc Diabetol 15 (2016) 53.

[5] C. Vasquez-Trincado, I. Garcia-Carvajal, C. Pennanen, V. Parra, J.A. Hill, B.A. Rothermel, S. Lavandero, Mitochondrial dynamics, mitophagy and cardiovascular disease, J Physiol 594(3) (2016) 509-25.

[6] E.P. Yu, M.R. Bennett, Mitochondrial DNA damage and atherosclerosis, Trends Endocrinol Metab 25(9) (2014) 481-7.

[7] A.J. Kattoor, N.V.K. Pothineni, D. Palagiri, J.L. Mehta, Oxidative Stress in Atherosclerosis, Curr Atheroscler Rep 19(11) (2017) 42.

[8] R. Singh, S. Devi, R. Gollen, Role of free radical in atherosclerosis, diabetes and dyslipidaemia: largerthan-life, Diabetes Metab Res Rev 31(2) (2015) 113-26.

[9] P.D. Ray, B.W. Huang, Y. Tsuji, Reactive oxygen species (ROS) homeostasis and redox regulation in cellular signaling, Cell Signal 24(5) (2012) 981-90.

[10] R. Stefanatos, A. Sanz, The role of mitochondrial ROS in the aging brain, FEBS Lett 592(5) (2018) 743-758.

[11] J. Van den Bossche, J. Baardman, N.A. Otto, S. van der Velden, A.E. Neele, S.M. van den Berg, R. Luque-Martin, H.J. Chen, M.C. Boshuizen, M. Ahmed, M.A. Hoeksema, A.F. de Vos, M.P. de Winther, Mitochondrial Dysfunction Prevents Repolarization of Inflammatory Macrophages, Cell Rep 17(3) (2016) 684-696.

[12] M. Xiao, H. Zhong, L. Xia, Y. Tao, H. Yin, Pathophysiology of mitochondrial lipid oxidation: Role of 4hydroxynonenal (4-HNE) and other bioactive lipids in mitochondria, Free Radic Biol Med 111 (2017) 316327. 
[13] D.A. Chistiakov, T.P. Shkurat, A.A. Melnichenko, A.V. Grechko, A.N. Orekhov, The role of mitochondrial dysfunction in cardiovascular disease: a brief review, Ann Med 50(2) (2018) 121-127.

[14]Nasiri-Ansari N, Dimitriadis GK, Agrogiannis G , Perrea D , Kostakis ID $₫$ Canagliflozin attenuates the progression of atherosclerosis and inflammation process in APOE knockout mice区Cardiovasc Diabetol. 2018 Jul 26;17(1):106.

[15] T. Yuan, T. Yang, H. Chen, D. Fu, Y. Hu, J. Wang, Q. Yuan, H. Yu, W. Xu, X. Xie, New insights into oxidative stress and inflammation during diabetes mellitus-accelerated atherosclerosis, Redox Biol 20 (2019) 247-260.

[16] L. Montenegro, M.N. Modica, L. Salerno, A.M. Panico, L. Crasci, G. Puglisi, G. Romeo, In Vitro Antioxidant Activity of Idebenone Derivative-Loaded Solid Lipid Nanoparticles, Molecules 22(6) (2017).

[17] S. Jaber, B.M. Polster, Idebenone and neuroprotection: antioxidant, pro-oxidant, or electron carrier?, J Bioenerg Biomembr 47(1-2) (2015) 111-8.

[18] P. Lin, J. Liu, M. Ren, K. Ji, L. Li, B. Zhang, Y. Gong, C. Yan, Idebenone protects against oxidized low density lipoprotein induced mitochondrial dysfunction in vascular endothelial cells via GSK3beta/betacatenin signalling pathways, Biochem Biophys Res Commun 465(3) (2015) 548-55.

[19] D.B. Zorov, M. Juhaszova, S.J. Sollott, Mitochondrial reactive oxygen species (ROS) and ROS-induced ROS release, Physiol Rev 94(3) (2014) 909-50.

[20] X. Ren, L. Ren, Q. Wei, H. Shao, L. Chen, N. Liu, Advanced glycation end-products decreases expression of endothelial nitric oxide synthase through oxidative stress in human coronary artery endothelial cells, Cardiovasc Diabetol 16(1) (2017) 52.

[21] S.W. Ballinger, C. Patterson, C.A. Knight-Lozano, D.L. Burow, C.A. Conklin, Z. Hu, J. Reuf, C. Horaist, R. Lebovitz, G.C. Hunter, K. Mclntyre, M.S. Runge, Mitochondrial integrity and function in atherogenesis, Circulation 106(5) (2002) 544-9.

[22] E. Yu, P.A. Calvert, J.R. Mercer, J. Harrison, L. Baker, N.L. Figg, S. Kumar, J.C. Wang, L.A. Hurst, D.R. Obaid, A. Logan, N.E. West, M.C. Clarke, A. Vidal-Puig, M.P. Murphy, M.R. Bennett, Mitochondrial DNA damage can promote atherosclerosis independently of reactive oxygen species through effects on smooth muscle cells and monocytes and correlates with higher-risk plaques in humans, Circulation 128(7) (2013) 702-12.

[23] S. Subramanian, B. Kalyanaraman, R.Q. Migrino, Mitochondrially targeted antioxidants for the treatment of cardiovascular diseases, Recent Pat Cardiovasc Drug Discov 5(1) (2010) 54-65.

[24] J. Tiefenbach, L. Magomedova, J. Liu, A.A. Reunov, R. Tsai, N.S. Eappen, R.A. Jockusch, C. Nislow, C.L. Cummins, H.M. Krause, Idebenone and coenzyme Q10 are novel PPARalpha/gamma ligands, with potential for treatment of fatty liver diseases, Dis Model Mech 11(9) (2018). 
[25] A. Yan, Z. Liu, L. Song, X. Wang, Y. Zhang, N. Wu, J. Lin, Y. Liu, Z. Liu, Idebenone Alleviates Neuroinflammation and Modulates Microglial Polarization in LPS-Stimulated BV2 Cells and MPTPInduced Parkinson's Disease Mice, Front Cell Neurosci 12 (2018) 529.

[26] C. Becker, K. Bray-French, J. Drewe, Pharmacokinetic evaluation of idebenone, Expert Opin Drug Metab Toxicol 6(11) (2010) 1437-44.

[27] S.M. Cardoso, C. Pereira, R. Oliveira, Mitochondrial function is differentially affected upon oxidative stress, Free Radic Biol Med 26(1-2) (1999) 3-13.

[28] V. Giorgio, M. Schiavone, C. Galber, M. Carini, T. Da Ros, V. Petronilli, F. Argenton, V. Carelli, M.J. Acosta Lopez, L. Salviati, M. Prato, P. Bernardi, The idebenone metabolite QS10 restores electron transfer in complex I and coenzyme Q defects, Biochim Biophys Acta Bioenerg 1859(9) (2018) 901-908.

[29] P. Yu-Wai-Man, D. Soiferman, D.G. Moore, F. Burte, A. Saada, Evaluating the therapeutic potential of idebenone and related quinone analogues in Leber hereditary optic neuropathy, Mitochondrion 36 (2017) 36-42.

[30] T. Kobayashi, K. Yoshida, M. Mitani, H. Torii, S. Tanayama, Metabolism of idebenone (CV-2619), a new cerebral metabolism improving agent: isolation and identification of metabolites in the rat and dog, $\mathrm{J}$ Pharmacobiodyn 8(6) (1985) 448-56.

[31] Z.R. Xu, J.Y. Li, X.W. Dong, Z.J. Tan, W.Z. Wu, Q.M. Xie, Y.M. Yang, Apple Polyphenols Decrease Atherosclerosis and Hepatic Steatosis in ApoE-/- Mice through the ROS/MAPK/NF-kappaB Pathway, Nutrients 7(8) (2015) 7085-105.

[32] N. Gueven, Idebenone for Leber's hereditary optic neuropathy, Drugs Today (Barc) 52(3) (2016) 17381.

[33] L.M. Fadda, H. Hagar, A.M. Mohamed, H.M. Ali, Quercetin and Idebenone Ameliorate Oxidative Stress, Inflammation, DNA damage, and Apoptosis Induced by Titanium Dioxide Nanoparticles in Rat Liver, Dose Response 16(4) (2018) 1559325818812188.

[34] J. Traba, S.S. Geiger, M. Kwarteng-Siaw, K. Han, O.H. Ra, R.M. Siegel, D. Gius, M.N. Sack, Prolonged fasting suppresses mitochondrial NLRP3 inflammasome assembly and activation via SIRT3-mediated activation of superoxide dismutase 2, J Biol Chem 292(29) (2017) 12153-12164.

[35] A.E. Kane, D.A. Sinclair, Sirtuins and $\mathrm{NAD}(+)$ in the Development and Treatment of Metabolic and Cardiovascular Diseases, Circ Res 123(7) (2018) 868-885.

[36] B. Sosnowska, M. Mazidi, P. Penson, A. Gluba-Brzozka, J. Rysz, M. Banach, The sirtuin family members SIRT1, SIRT3 and SIRT6: Their role in vascular biology and atherogenesis, Atherosclerosis 265 (2017) 275-282. 
[37] J. Wu, Z. Zeng, W. Zhang, Z. Deng, Y. Wan, Y. Zhang, S. An, Q. Huang, Z. Chen, Emerging role of SIRT3 in mitochondrial dysfunction and cardiovascular diseases, Free Radic Res 53(2) (2019) 139-149.

[38] M.L. Chen, X.H. Zhu, L. Ran, H.D. Lang, L. Yi, M.T. Mi, Trimethylamine-N-Oxide Induces Vascular Inflammation by Activating the NLRP3 Inflammasome Through the SIRT3-SOD2-mtROS Signaling Pathway, J Am Heart Assoc 6(9) (2017).

[39] I. Salvatori, C. Valle, A. Ferri, M.T. Carri, SIRT3 and mitochondrial metabolism in neurodegenerative diseases, Neurochem Int 109 (2017) 184-192.

[40] S. Karnewar, S.B. Vasamsetti, R. Gopoju, A.K. Kanugula, S.K. Ganji, S. Prabhakar, N. Rangaraj, N. Tupperwar, J.M. Kumar, S. Kotamraju, Mitochondria-targeted esculetin alleviates mitochondrial dysfunction by AMPK-mediated nitric oxide and SIRT3 regulation in endothelial cells: potential implications in atherosclerosis, Sci Rep 6 (2016) 24108.

[41] A.E. Dikalova, H.A. Itani, R.R. Nazarewicz, W.G. McMaster, C.R. Flynn, R. Uzhachenko, J.P. Fessel, J.L. Gamboa, D.G. Harrison, S.I. Dikalov, Sirt3 Impairment and SOD2 Hyperacetylation in Vascular Oxidative Stress and Hypertension, Circ Res 121(5) (2017) 564-574.

[42] M. Haneklaus, L.A. O'Neill, NLRP3 at the interface of metabolism and inflammation, Immunol Rev 265(1) (2015) 53-62.

[43] N. Song, T. Li, Regulation of NLRP3 Inflammasome by Phosphorylation, Front Immunol 9 (2018) 2305.

[44] L. Minutoli, D. Puzzolo, M. Rinaldi, N. Irrera, H. Marini, V. Arcoraci, A. Bitto, G. Crea, A. Pisani, F. Squadrito, V. Trichilo, D. Bruschetta, A. Micali, D. Altavilla, ROS-Mediated NLRP3 Inflammasome Activation in Brain, Heart, Kidney, and Testis Ischemia/Reperfusion Injury, Oxid Med Cell Longev 2016 (2016) 2183026.

[45] E.K. Jo, J.K. Kim, D.M. Shin, C. Sasakawa, Molecular mechanisms regulating NLRP3 inflammasome activation, Cell Mol Immunol 13(2) (2016) 148-59.

[46] A. Grebe, F. Hoss, E. Latz, NLRP3 Inflammasome and the IL-1 Pathway in Atherosclerosis, Circ Res 122(12) (2018) 1722-1740.

[47] Z. Hoseini, F. Sepahvand, B. Rashidi, A. Sahebkar, A. Masoudifar, H. Mirzaei, NLRP3 inflammasome: Its regulation and involvement in atherosclerosis, J Cell Physiol 233(3) (2018) 2116-2132.

[48] R. Wang, Y. Wang, N. Mu, X. Lou, W. Li, Y. Chen, D. Fan, H. Tan, Activation of NLRP3 inflammasomes contributes to hyperhomocysteinemia-aggravated inflammation and atherosclerosis in apoE-deficient mice, Lab Invest 97(8) (2017) 922-934. 
[49] Neuhofer A, Wernly B, Leitner L, Sarabi A, Sommer NG, Staffler G, Zeyda M, Stulnig TM $\unrhd$ An accelerated mouse model for atherosclerosis and adipose tissue inflammation $\mathbb{}$ Cardiovasc Diabetol. 2014 Jan 17;13:23.

[50] J.W. Yu, M.S. Lee, Mitochondria and the NLRP3 inflammasome: physiological and pathological relevance, Arch Pharm Res 39(11) (2016) 1503-1518.

[51] T. Sho, J. Xu, Role and mechanism of ROS scavengers in alleviating NLRP3-mediated inflammation, Biotechnol Appl Biochem 66(1) (2019) 4-13.

[52] J. Tschopp, K. Schroder, NLRP3 inflammasome activation: The convergence of multiple signalling pathways on ROS production?, Nat Rev Immunol 10(3) (2010) 210-5.

[53] J.B. Perry, G.N. Davis, M.E. Allen, M. Makrecka-Kuka, M. Dambrova, R.W. Grange, S.R. Shaikh, D.A. Brown, Cardioprotective effects of idebenone do not involve ROS scavenging: Evidence for mitochondrial complex I bypass in ischemia/reperfusion injury, J Mol Cell Cardiol 135 (2019) 160-171.

\section{Supplementary File Legend}

Figure 1S. COG/KOG functional classification chart of differentially expressed proteins. COG/KOG functional classification chart of differentially expressed proteins mainly involved in (C) energy production and conversion; (E) amino acid transport and metabolism; (I) lipid transport and metabolism; (R) genera function prediction; and $(\mathrm{T})$ signal transduction mechanisms.

\section{Figures}



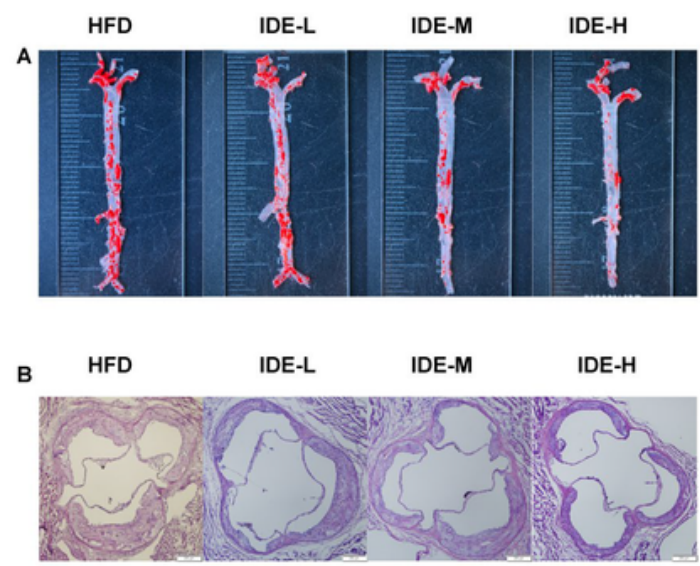

c

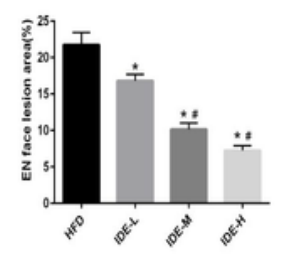

D

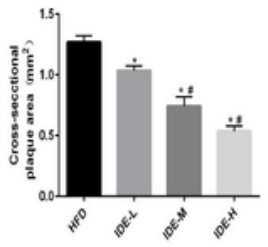

\section{Figure 2}

Idebenone suppresses the formation of atherosclerotic plaques in apoE-/- mice. (A) Representative images of en face Oil-Red $\mathrm{O}$ staining of aortas from the four groups of mice. (B) Quantitative analysis of en face aortic lesions expressed as the percentage of lesion area relative to the total aortic area. (C) Representative images of cross-sectional aortic root lesions by H\&E staining. (D) Quantitative analysis of 
cross-sectional plaque areas in the aortic roots. Data are expressed as the mean $\pm S E M .{ }^{*}<0.05$ vs. HFD, \#P<0.05 vs. IDE-L. HFD: high fat diet, IDE: Idebenone, L: low-dose, M: medium-dose, H: high-dose.

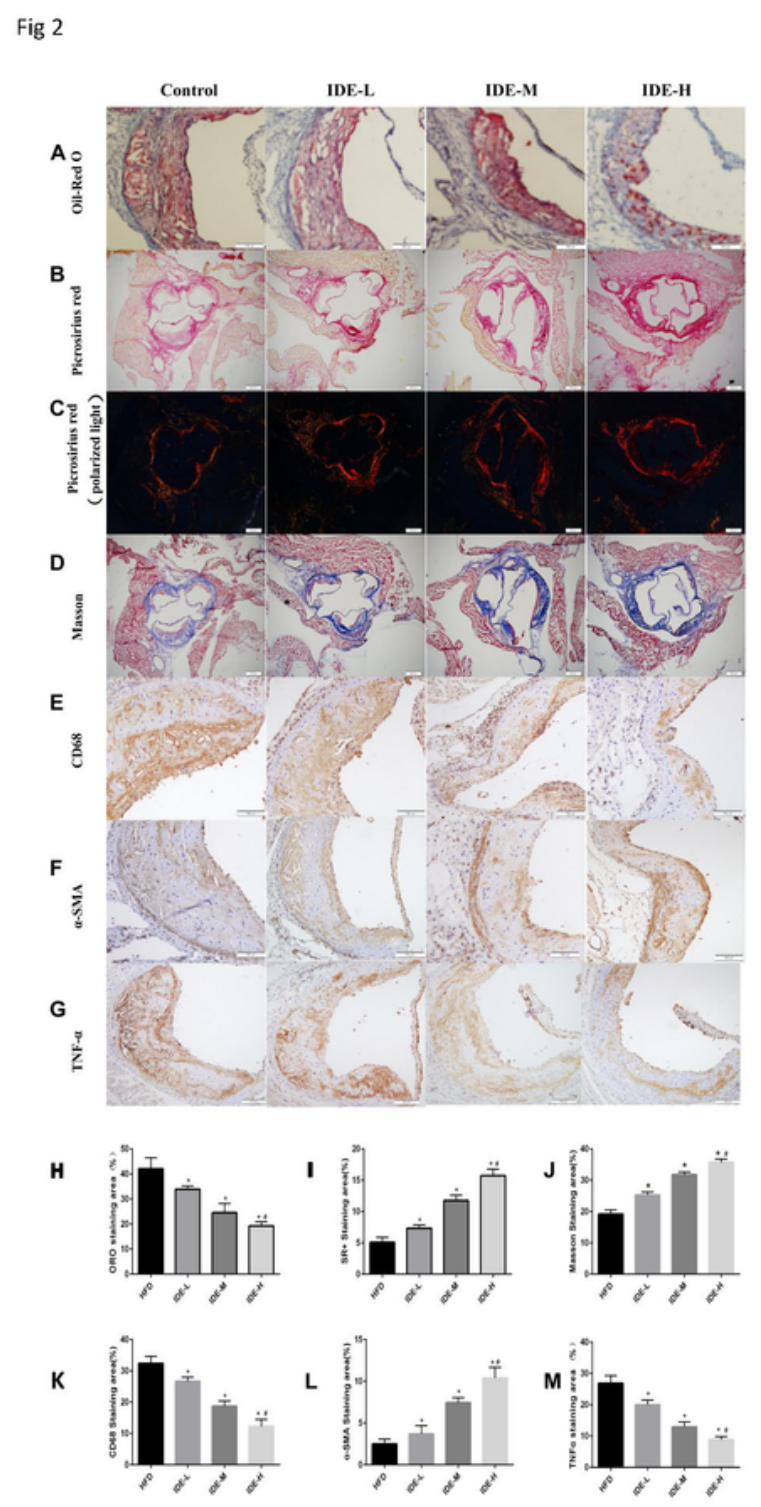

\section{Figure 3}

Idebenone treatment increases the stability of atherosclerotic plaques in apoE-/- mice. (A) Representative image of lipid deposition in the four treatment groups by Oil-Red $O$ staining. (B) Representative image of the fibrous cap thickness by picrosirius red staining. (C) Representative image of the fibrous cap 
thickness by picrosirius red staining, as illuminated with polarized light. (D) Representative image of collagen fibers by Masson staining. (E) Representative immunohistochemical CD68 staining of plaque macrophage content. (F) Representative immunohistochemical a-SMA staining of plaque smooth muscle cell content. (G) Representative immunohistochemical TNF-a staining of plaque smooth muscle cell content. (H) Quantification of lipid deposition in the four treatment groups by Oil-Red $\mathrm{O}$ staining. (I) Quantification of the fibrous cap thickness by picrosirius red staining, as illuminated with polarized light in the four groups of mice. $(\mathrm{J})$ Quantification of plaque collagen content by Masson staining in the four groups of mice. (K) Quantification of plaque macrophage content in the four groups of mice. $(\mathrm{L})$ Quantification of plaque smooth muscle cell content in the four groups of mice.(M) Quantification of plaque TNF-a content in the four groups of mice. Data are expressed as the mean $\pm S E M$. ${ }^{*} P<0.05$ vs. HFD. \#P<0.05 vs. IDE-L. HFD: high fat diet, IDE: Idebenone,L: low-dose, M: medium-dose, H: high-dose. 
Fig 3
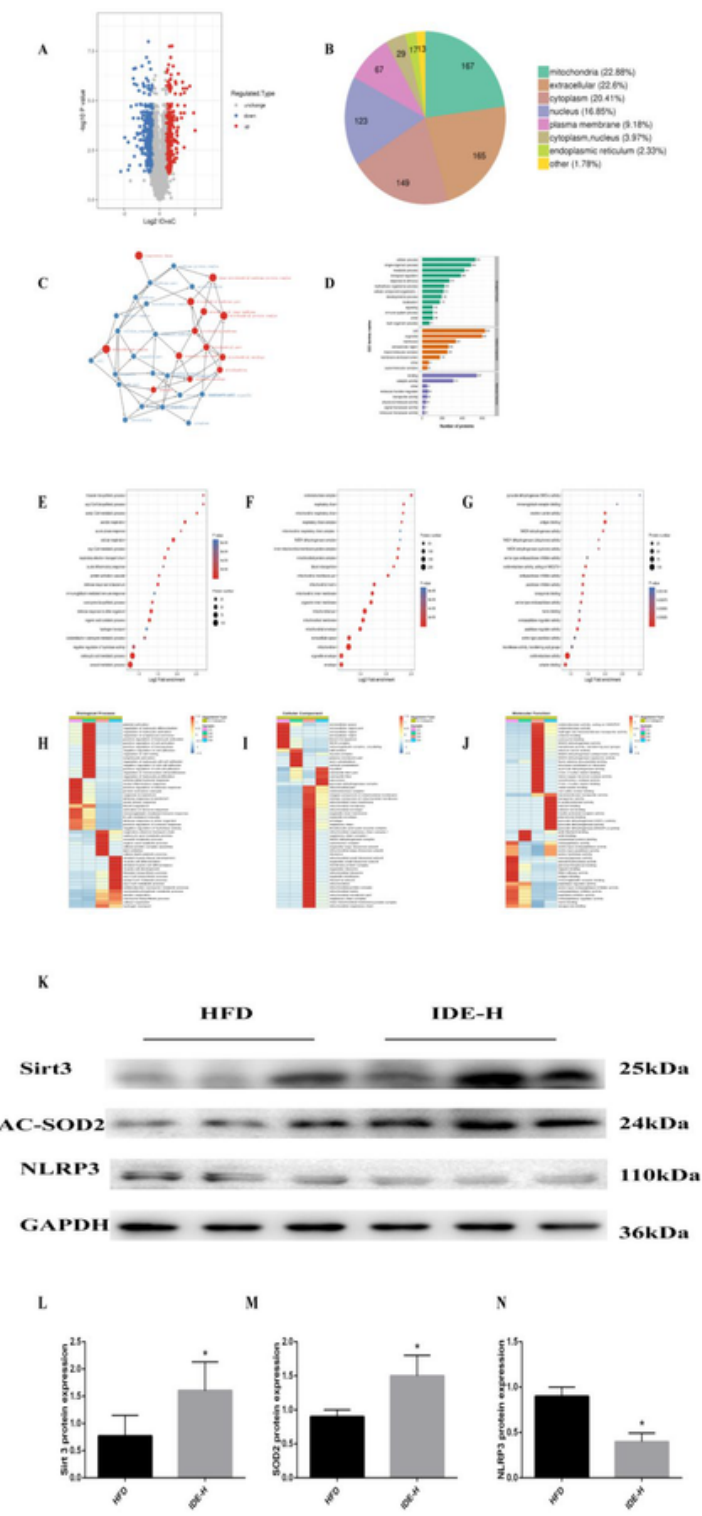

\section{Figure 5}

Proteomic analysis between the HFD group $(n=3)$ and the idebenone-H group $(n=3)$. (A) Volcano plot of differentially expressed proteins. (B) Subcellular localization chart of differentially expressed proteins. (C) DAG (Directed Acyclic Graph) of each GO category for differentially expressed proteins. (D) Statistical distribution chart of differentially expressed proteins under each GO category. (E-G) GO enrichment bubble plot of differentially expressed proteins in three categories. (H-J) comprehensive heatmap for 
cluster analysis of the enrichment patterns of GO functional categories; (K) Validation of screened differential proteins: representative Western blot image of SIRT3, AC-SOD2, and NLRP3. (L) Quantification of the protein ratio of SIRT3/GAPDH between the HFD group and the idebenone-H group. (M) Quantification of the protein ratio of AC-SOD2/GAPDH between the HFD group and the idebenone-H group. (N) Quantification of the protein ratio of NLRP3/GAPDH between the HFD group and the idebenone-H group. Data are expressed as the mean \pm SEM. ${ }^{*} P<0.05$ vs. HFD. HFD: high fat diet, IDE: Idebenone, L: low-dose, M: medium-dose, H: high-dose , AC-SOD2: acetylated SOD2.

Fig 4

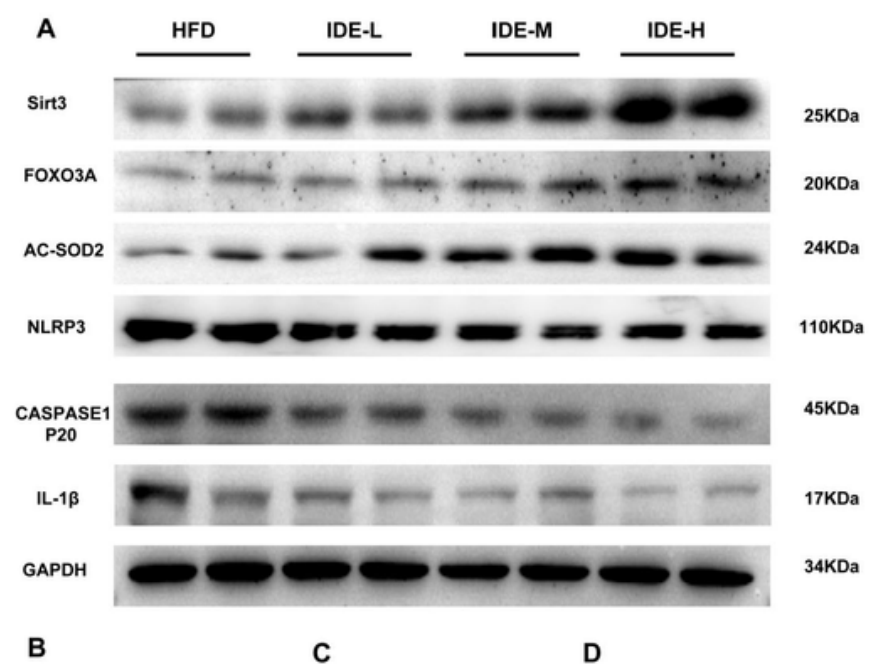

B
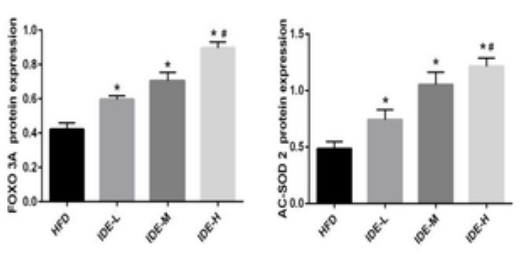

$E$

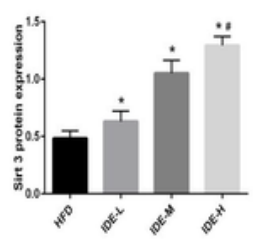

$\mathbf{F}$

G
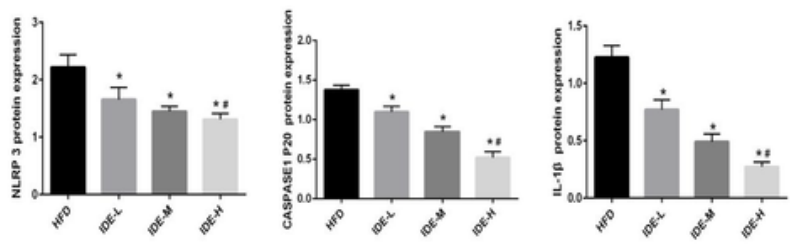


\section{Figure 8}

Idebenone activates the SIRT3-SOD2-mtROS pathway in apoE-/- mice. (A) Representative Western blot image of SIRT3, FOXO3A, AC-SOD2, NLRP3, Caspase-1(p20) , and IL-1 ßin the four different treatment groups with apoE-/- mice. Quantification of the expression of SIRT3 (B), FOXO3A (C), AC-SOD2 (D), NLRP3 (E), Caspase-1 (p20) (F), and IL-1 $\beta(G)$ in the four different treatment groups with apoE-/- mice. Data are expressed as the mean \pm SEM. ${ }^{*} P<0.05$ vs. HFD. \#P<0.05 vs. IDE-L. HFD: high fat diet, IDE: Idebenone, L: low-dose, M: medium-dose, H: high-dose, AC-SOD2: acetylated SOD2.

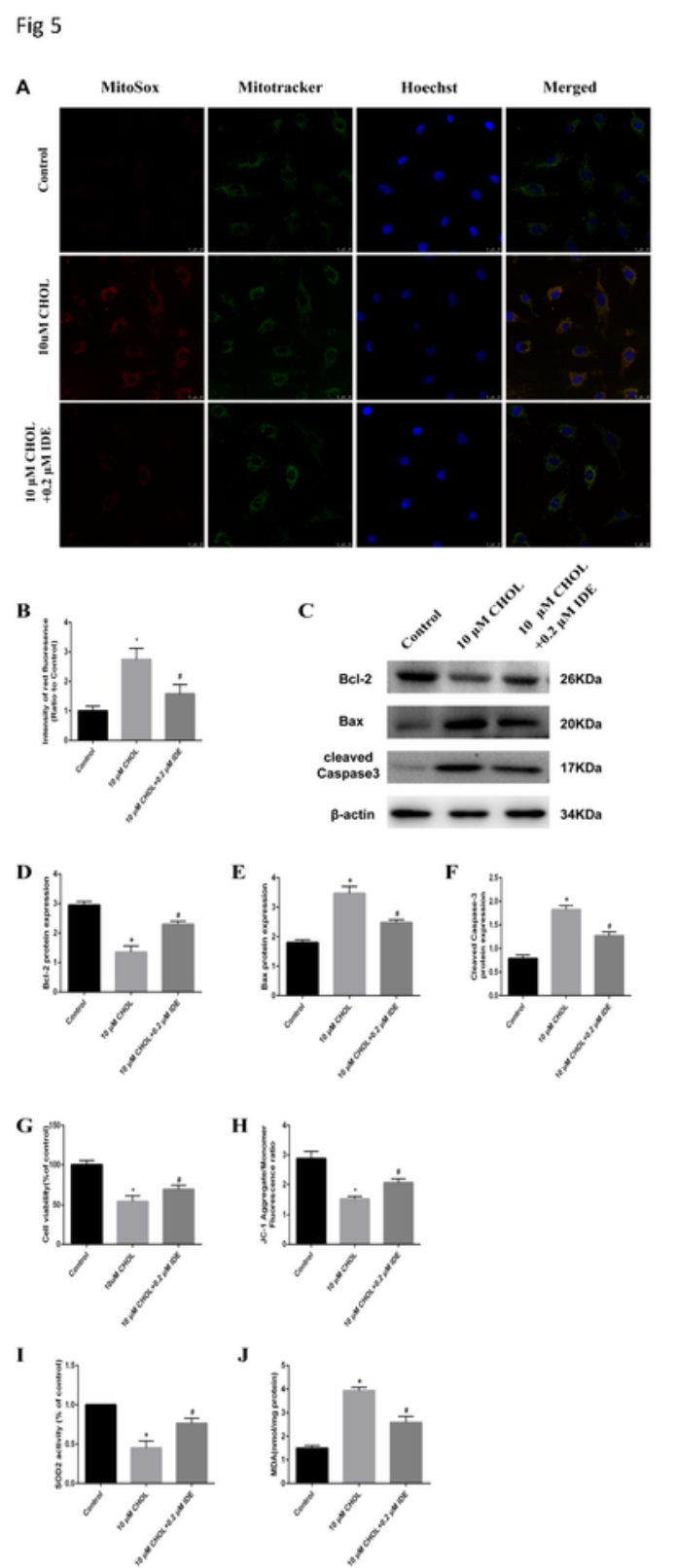




\section{Figure 10}

Idebenone protects against endothelial cell damage and inhibits the production of mtROS in HUVECs treated with cholesterol. (A) Representative fluorescence image in each group by MitoSox and Mitotracker staining. (B) Quantitative analysis of intensity of red fluorescence by MitoSox staining. (C) Representative Western blot image of Bcl-2, Bax, Cleaved Caspase-3 in the cholesterol-induced HUVECs damage model and idebenone treatment group. Quantified expression of Bcl-2 (D), Bax (E), Cleaved Caspase-3 (F) in the cholesterol-induced HUVECs damage model and idebenone treatment group. (G) CCK8 detects the inhibition of HUVEC proliferation in different treatment groups. $(\mathrm{H}) \mathrm{JC}-1$ staining for detecting the ratio of aggregate-to-monomer fluorescence of each group. ( $I$ and $J$ ) The effects of idebenone on MDA and SOD2 activity were measured by using ELISA. Representative images were from three independent experiments. Data are expressed as the mean \pm SEM. ${ }^{*} \mathrm{P}<0.05$ vs. control.; $\# \mathrm{P}<0.05$ vs. $10 \mu \mathrm{M}$ CHOL. CHOL: cholesterol , AC-SOD2: acetylated SOD2. 
Fig 6

A

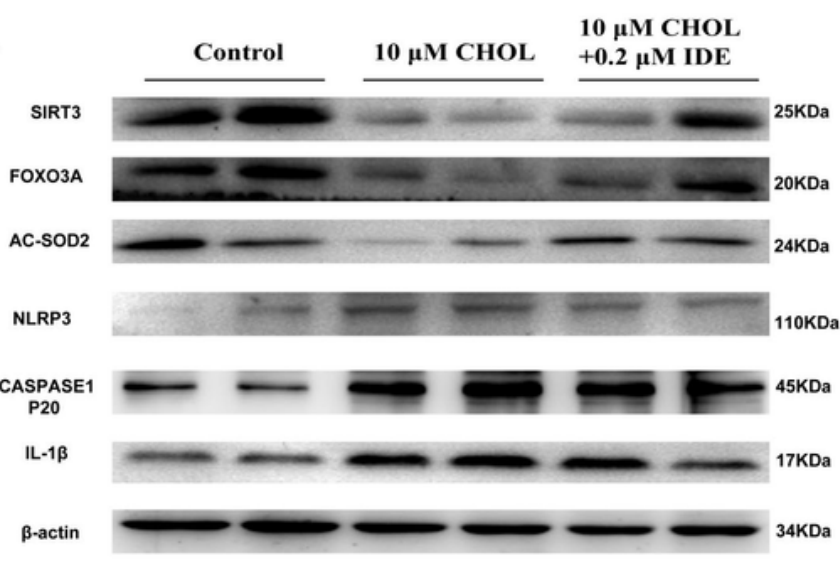

B

C

D
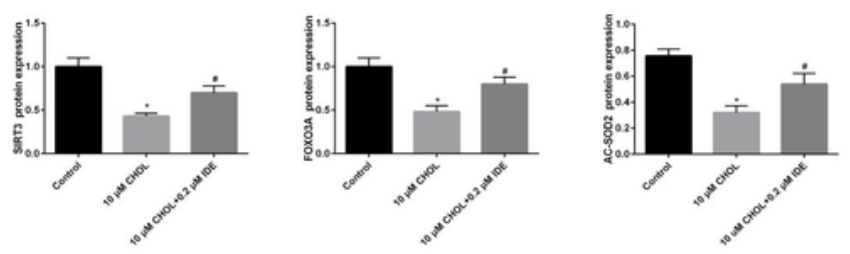

E

$\mathbf{F}$

G
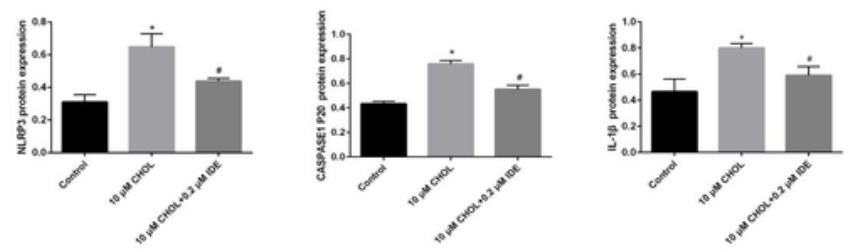

\section{Figure 12}

Idebenone inhibits cholesterol-induced injury of HUVECs via modulation of SIRT3 activation. (A) Representative Western blot image of SIRT3, FOXO3A, AC-SOD2, NLRP3, Caspase-1 (p20), and IL-1 $\beta$ in the cholesterol-induced HUVECs damage model and idebenone treatment group. Quantified expression of SIRT3 (B), FOX03A (C), AC-SOD2 (D), NLRP3 (E), Caspase-1 (p20) (F), IL-1ß(G) in the cholesterol-induced HUVECs damage model and idebenone treatment group. Representative images were from three 
independent experiments. Data are expressed as the mean \pm SEM. ${ }^{*} \mathrm{P}<0.05$ vs. control; $\# \mathrm{P}<0.05$ vs. $10 \mu \mathrm{M}$ CHOL. CHOL: cholesterol, IDE: idebenone, AC-SOD2: acetylated SOD2.

\section{Supplementary Files}

This is a list of supplementary files associated with this preprint. Click to download.

- FigureS1.pdf

- FigureS1.pdf 